\title{
Thrombopoietin concentrations are low in patients with cirrhosis and thrombocytopenia and are restored after orthotopic liver transplantation
}

\author{
J Goulis, T N Chau, S Jordan, A B Mehta, A Watkinson, K Rolles, A K Burroughs
}

Department of Liver Transplantation and Hepatobiliary Medicine, Royal Free Hospital, Pond Street, London NW3 2QG, UK J Goulis

T N Chau

K Rolles

A K Burroughs

Department of Haematology, Royal

Free Hospital, Pond

Street, London

NW3 2QG, UK

$S$ Jordan

A B Mehta

Department of

Radiology, Royal Free

Hospital, Pond Street,

London NW3 2QG, UK

A Watkinson

Correspondence to:

Dr Burroughs.

Accepted for publication 18 December 1998

\begin{abstract}
Background-Thrombocytopenia in cirrhotic patients may be due to deficient production of thrombopoietin.

Aims-To determine the relation between thrombopoietin and thrombocytopenia in cirrhotic patients before and after orthotopic liver transplantation.

Methods-Thrombopoietin concentrations and platelet counts were measured in 43 cirrhotic patients and 21 normal controls and serially for 14 days after transplantation in 23/43 patients.

Results -27 of the 43 patients had thrombocytopenia (platelet count less than $120 \times$ $10^{9} / 1$; group 1) whereas 16 patients had normal platelet count (group 2). Thrombopoietin concentrations were lower in group 1 than in group $2(92.5(20.3-286.3)$ $v 226.6(30.1-848.3) \mathrm{pg} / \mathrm{ml}, \mathrm{p}=0.003)$ and normal controls $(92.5(20.3-286.3) v 158.3$ (22.5-232.9) pg/ml, $\mathrm{p}=0.028)$. Posttransplantation thrombopoietin concentrations increased with a peak at day 5 . The rise was significant in patients with low pretransplantation platelet count $(89.1$ (21.29-247.6) to $545.1(66.2-2569) \mathrm{pg} / \mathrm{ml}$; $\mathrm{n}=16, \mathrm{p}=0.001$ ) but not in those with normal platelet count (262.8 (30.1-848.3) to 315.1 (114-954.6) $\mathrm{pg} / \mathrm{ml} ; \mathrm{n}=7, \mathrm{p}=0.47)$. No correlation was found pretransplantation between spleen volume and platelet count $(r=-0.11, p=0.6)$ or thrombopoietin concentrations $(r=-0.04, p=0.8)$. However, pretransplantation thrombopoietin concentrations correlated with platelet count $(r=0.47, p=0.0015)$, whereas an inverse correlation was found between peak thrombopoietin concentrations and nadir platelet count $(r=-0.41 \quad p=0.049)$ posttransplantation.

Conclusions-Inadequate thrombopoietin production may contribute to cirrhotic thrombocytopenia. Thrombopoietin production is restored after liver transplantation leading to the resolution of thrombocytopenia. (Gut 1999;44:754-758)
\end{abstract}

Keywords: platelets; thrombocytopenia; thrombopoietin; cirrhosis; orthotopic liver transplantation

Thrombocytopenia is one of the most frequent haematological abnormalities in patients with cirrhosis and portal hypertension. It is generally considered to be due to the increased sequestration and destruction of platelets in the enlarged spleen which was defined as "hypersplenism". ${ }^{2}$ However, it has been shown that only a few patients with advanced liver disease and thrombocytopenia respond with an increase in bone marrow production of platelets. ${ }^{3}$ Moreover portal decompression procedures, either by surgical shunts or transjugular intrahepatic portosystemic shunts (TIPS), have not led to a consistent rise in platelet count. ${ }^{4-7}$ The only procedure which definitively resolves the thrombocytopenia of liver disease is orthotopic liver transplantation (OLT) ${ }^{8}$

Thrombopoietin (TPO) was recently cloned and identified as the primary cytokine involved in the maturation of megakaryocytes and formation of platelets. ${ }^{9}{ }^{10}$ Serum TPO concentrations are inversely related to platelet concentrations in patients with haematopoietic disorders characterised by decreased megakaryocytes in bone marrow. ${ }^{11}$ The level of expression of mRNA for TPO is high in the liver indicating that this is the main source of its synthesis. $^{91012}$ Thus it has been proposed that deficiency of TPO production could be responsible for the thrombocytopenia in cirrhosis.

Serum TPO concentrations have been measured previously in patients with liver disease with different results due mainly to a varied study population (cirrhotic or noncirrhotic patients, cirrhotic patients with or without thrombocytopenia, different severity of liver disease, etc.) and in the limitations of the assays used. ${ }^{13-15}$ Although two previous studies have reported that TPO rises after OLT, ${ }^{16}{ }^{17}$ the TPO concentrations before OLT were undetectable because of the poor sensitivity of the assay, so that the relation to platelet number could not be established.

The aim of our study was to determine the relation between TPO and thrombocytopenia in consecutive cirrhotic patients with end stage liver disease, some of whom subsequently had OLT. Our hypothesis was that inadequate TPO production contributes to the pathogenesis of thrombocytopenia of severe liver disease, and that there would be a correlation between low platelet count and low TPO concentrations.

Abbreviations used in this paper: DIC, disseminated intravascular coagulation; HBV, hepatitis $B$ virus; $\mathrm{HCV}$, hepatitis $\mathrm{C}$ virus; OLT, orthotopic liver transplantation; TPO, thrombopoietin; TIPS, transjugular intrahepatic portosystemic shunts; CT, computed tomography. 
Table 1 Demographic and laboratory characteristics of patients with (group 1) and without (group 2) thrombocytopenia

\begin{tabular}{lll}
\hline & Group 1 (n=27) & Group 2 $(n=16)$ \\
\hline Age $(\mathrm{y})^{\star}$ & $53(13)$ & $46(13)$ \\
Sex $(\mathrm{M} / \mathrm{F})$ & $15 / 12$ & $11 / 5$ \\
Cause of cirrhosis & & \\
$\quad$ Viral hepatitis (B and C) (\%) & $10(37)$ & $2(12.5)$ \\
$\quad$ Viral hepatitis and alcohol (\%) & $2(7.5)$ & $4(25)$ \\
Alcohol $(\%)$ & $7(26)$ & $6(37.5)$ \\
PBC/PSC (\%) & $5(18.5)$ & $4(25)$ \\
Other $(\%)$ & $3(11)$ & $33(8)$ \\
Albumin $(\mathrm{g} / \mathrm{l})^{\star}$ & $31(7)$ & $20(7)$ \\
Prothrombin time $(\mathrm{s})^{\star}$ & $26(14)$ & $85(37)$ \\
Bilirubin $(\mu \mathrm{mol} / 1)^{\star}$ & $155(37)$ & $8.5(1.0)$ \\
WBC count $\left(\times 10^{3} / \mathrm{mm}^{3}\right)^{\star}$ & $6.2(0.7)$ & $230(87)$ \\
Platelet count $\left(\times 10^{3} / \mathrm{mm}^{3}\right)^{\star}$ & $67(30)$ & \\
\hline
\end{tabular}

«Mean (SD).

PBC, primary biliary cirrhosis; PSC, primary sclerosing cholangitis; WBC, white blood cell.

\section{Patients and methods}

PATIENT POPULATION

Platelet counts and serum TPO concentrations were measured in 43 patients with cirrhosis of the liver diagnosed by histological evaluation of hepatic biopsy specimens. Patients with hepatocellular carcinoma and disseminated intravascular coagulation (DIC) were excluded from the study.

Twenty three of the 43 patients underwent OLT because of end stage liver disease. These patients received an $\mathrm{ABO}$ matched cadaver liver and the standard immunosuppressive regimen consisted of tacrolimus or cyclosporine, azathioprine, and corticosteroids. All 23 patients who underwent OLT had a generally uneventful post-transplantation period without serious complications. Liver biopsies were performed by protocol seven days after transplantation.

Serum samples for TPO measurement were obtained from the 23 patients who had OLT, on the day before OLT, and on days $1,3,5,7$, 10 , and 14 after OLT. One random serum sample was also taken from the 20 cirrhotic patients who did not undergo OLT. Platelet counts were measured on the day of TPO measurement for the non-transplanted patients and daily for all transplanted patients until discharge. The size of the spleen was measured pretransplantation by helical computed tomography (CT), using the spleen index. This was calculated by multiplication of the length of the longitudinal and transverse axes, the latter defined as the maximal width on the CT image.

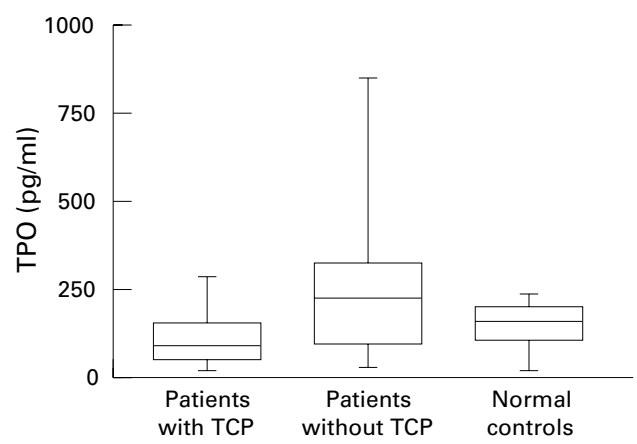

Figure 1 Baseline TPO concentrations in cirrhotic patients with or without thrombocytopenia (TCP) and normal controls. Boxes represent the $50 \%$ percentile of the values; the inside line represents the median value.
According to baseline platelet count all the cirrhotic patients were divided into two groups: group 1 consisted of patients with thrombocytopenia (platelet count not more than $120 \times$ $10^{9} / 1$ ) and group 2 patients without thrombocytopenia (platelet count greater than $120 \times$ $\left.10^{9} / 1\right)$. The cut off point of $120 \times 10^{9} / 1$ was used in accordance with recent literature. ${ }^{17}$ In addition serum TPO concentrations and platelet counts were measured in 21 normal individuals (group 3).

THROMBOPOIETIN ASSAY

TPO was measured in serum samples with an enzyme linked immunosorbent assay (ELISA; R\&D Systems, Minneapolis, Minnesota, USA), which uses the quantitative sandwich type technique. ELISA plates were precoated with murine monoclonal antibody against TPO. Standards (recombinant human TPO in a buffered protein base with preservative, lyophilised) and samples were incubated on the plates for three hours. Bound TPO was detected using monoclonal antibody against TPO conjugated to horseradish peroxidase. Plates were washed between steps. The optical density of each well was determined using a microplate reader set to $450 \mathrm{~nm}$ within 30 minutes. Samples were stored at $-70^{\circ} \mathrm{C}$ until analysed. All samples were assayed in duplicate. The lower detection limit of the assay was $20 \mathrm{pg} / \mathrm{ml}$. The intra-assay variation was $7.5 \%$ and the interassay variation $10.5 \%$.

\section{STATISTICAL ANALYSIS}

Results are presented as mean (SD) if quantitative variables were normally distributed and as median (range) if they were not. Serum TPO concentrations are not normally distributed. Therefore the significance of the differences between group means of TPO was assessed by the Mann-Whitney $U$ test. The Wilcoxon signed rank test was used for the analysis of paired samples. The Spearman's rank correlation coefficient was used to examine the association between the parameters. A $p$ value less than 0.05 was considered statistically significant.

\section{Results}

The study population comprised 43 patients. The cause of cirrhosis was alcoholic in 11 cases, viral hepatitis (due to hepatitis $\mathrm{B}$ virus (HBV) or HCV) in 12, viral hepatitis and alcohol in two, primary biliary cirrhosis in seven, primary sclerosing cholangitis in four, Wilson's disease in one, Budd-Chiari syndrome in one, cryptogenic in two, and other causes in three. Table 1 shows the demographic and laboratory characteristics of the patients.

There were 27 patients in group 1 and 16 in group 2. Baseline platelet counts (mean (SD)) were $66(30) \times 10^{9} / 1$ in patients from group 1 and $230(87) \times 10^{9} / 1$ in those from group 2 $(\mathrm{p}<0.0001$; table 1$)$. Patients who had OLT had an uneventful post-transplantation period without serious complications. The protocol biopsies done on the seventh day after transplantation showed no features of acute 
Table 2 Follow up of patients with (group 1) and without (group 2) thrombocytopenia after $O L T$

\begin{tabular}{lll}
\hline & Group 1 & Group 2 \\
\hline Pre-OLT TPO $(\mathrm{pg} / \mathrm{ml})^{\star}$ & $89.1(21.3-247.6)$ & $262.8(30.1-848.3)$ \\
Days to peak TPO† & $4.5(2.8)$ & $5.5(3.3)$ \\
Peak TPO $(\mathrm{pg} / \mathrm{ml})^{\star}$ & $545.1(66.2-2569)$ & $315.1(114-954.6)$ \\
Day of nadir platelet count $\dagger$ & $4.6(1.4)$ & $4.7(1.1)$ \\
Days to normal platelet count $\dagger\left(>120 \times 10^{9} / 1\right)$ & $11(3)$ & $9(2)$
\end{tabular}

${ }^{\star}$ Median (range).

†Mean (SD).

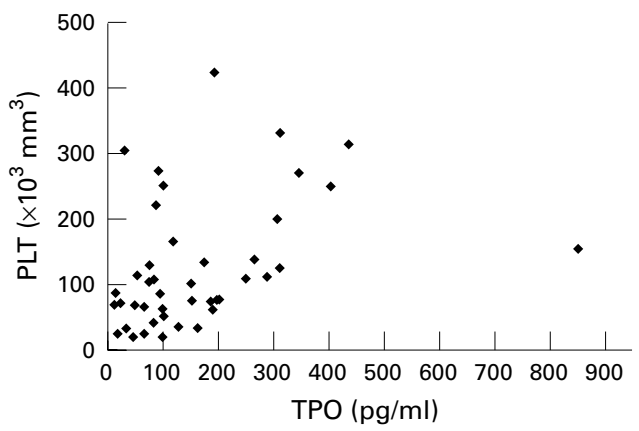

Figure 4 Correlation between TPO concentrations and platelet (PLT) count at baseline.

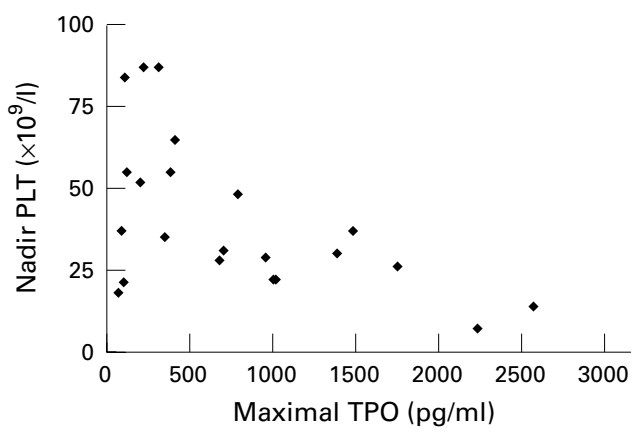

Figure 5 Correlation between maximum TPO and nadir platelet (PLT) count post-OLT.

centrations four to six days after peak TPO (table 2), while TPO concentrations returned to normal values. $\mathrm{ml}, \mathrm{p}=0.003)$ and to normal controls (92.5 (20.3-286.3) versus $158.3(22.5-232.9) \mathrm{pg} /$ $\mathrm{ml}, \mathrm{p}=0.025)$. TPO concentrations in patients with normal platelet count did not differ from those in normal controls $(\mathrm{p}=0.11$; fig 1$)$.

Thrombopoietin concentrations increased from day 1 after OLT and reached a peak on day 5. The rise in TPO concentrations was significant in patients with low baseline platelet count (89.1 (21.29-247.6) to 545.1 (66.22569) $\mathrm{pg} / \mathrm{ml}, \mathrm{p}=0.001)$, but not in transplanted patients with normal baseline platelet count (262.8 (30.1-848.3) to 315.1 (114-954.6) $\mathrm{pg} / \mathrm{ml}, \mathrm{p}=0.47$; table 2). Platelet counts decreased further from baseline in all the transplanted patients in the first days after OLT and reached a nadir concentration on day 5 post-OLT, which was the day of the peak TPO concentrations. Platelet counts subsequently increased and returned to normal con-

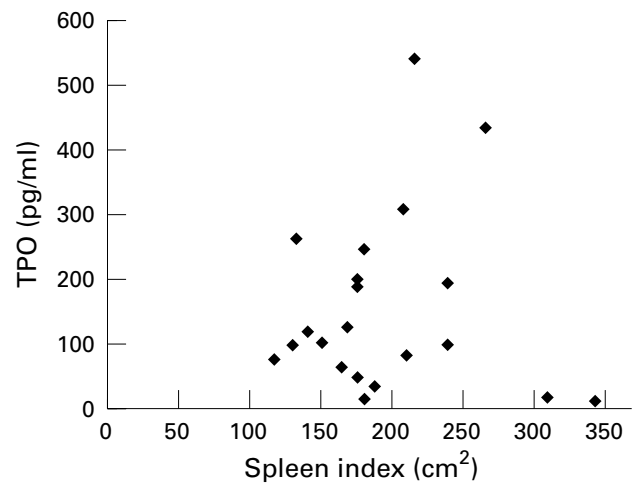

Figure 3 Correlation between TPO concentration and spleen index pre-OLT.
RELATION BETWEEN PLATELET COUNT AND TPO CONCENTRATIONS

Pre-OLT there was no correlation between spleen volume (as determined by spleen index) and platelet count $(r=-0.11, \mathrm{p}=0.6$; fig 2$)$ or TPO concentrations $(r=-0.04, \mathrm{p}=0.8$; fig 3$)$. However, there was a significant correlation between TPO concentrations before OLT and platelet count $(r=0.47, \mathrm{p}=0.001$; fig 4$)$. After transplantation there was an inverse correlation between peak TPO concentrations and nadir platelet count ( $r=-0.41, \mathrm{p}=0.049$; fig 5$)$.

There was no statistical correlation between peak TPO concentrations after OLT and cold ischaemia time $(r=0.1, \mathrm{p}=0.62)$, prothrombin time $(r=-0.04, p=0.9)$, or other parameters of liver function: maximum serum aspartate aminotransferase $(r=-0.001, \mathrm{p}=0.98)$, maximum serum alanine aminotransferase $(r=-0.04, \mathrm{p}=0.85)$, and maximum bilirubin $(r=-0.13, \mathrm{p}=0.6)$. Finally, there was no difference in the TPO concentrations between patients with $(n=15)$ or without histological rejection $(\mathrm{n}=8)(412.9 \quad(66.2-2569)$ versus $496.2(85.1-1746) \mathrm{pg} / \mathrm{ml}, \mathrm{p}=0.97)$.

\section{Discussion}

Serum TPO concentrations were measured in patients with end stage cirrhosis who were on concentrations were significantly lower in cirrhotic patients with thrombocytopenia (platelet count not more than $120 \times 10^{9} / 1$ ) compared with those with normal platelet count (greater than $\left.120 \times 10^{9} / 1\right)$. There was also the waiting list for liver transplantation. TPO 
a sharp rise in TPO concentrations in cirrhotics with thrombocytopenia starting from the first day after liver transplantation and reaching a peak on the fifth day. The ELISA used in this study (R\&D Systems, Minneapolis, Minnesota, USA) was highly sensitive, having a lower detection limit of $20 \mathrm{pg} / \mathrm{ml}$. This overcame the limitations of the assays in previous studies ${ }^{16}{ }^{17}$ and permitted measurement of TPO concentrations in the serum of all cirrhotic patients and normal subjects.

The hypothesis that liver could produce factors capable of stimulating thrombopoiesis is not new. Several years ago, it was reported that in rodent models, partial hepatectomy results in thrombocytopenia. ${ }^{18}$ The identification of the liver as the main source of TPO production $^{9} 0^{12}$ has led to the suggestion that deficiency of this cytokine contributes to the thrombocytopenia of end stage liver disease. Previous studies in which TPO was measured in patients with liver cirrhosis have shown inconsistent results, due mainly to differences in the study populations. ${ }^{13-15} 19$ The main problem with the interpretation of these studies is that classification of patients in the different study groups was performed independently of the platelet count. Although cirrhotics had generally lower platelet counts than their control populations used as comparison, there were patients with normal platelet counts in the cirrhotic groups. ${ }^{13-15}$ Hence the concentrations of TPO in cirrhotic patients with thrombocytopenia, which is the subgroup of interest, was never clear. Furthermore, the differences in the severity of liver disease are not generally taken into account.

Our population consisted exclusively of patients with end stage cirrhosis (with no Child class A patients) who were on the waiting list for liver transplantation. Over half of them subsequently underwent liver transplantation, and the rest were still waiting. Moreover we separated patients with cirrhosis into those with and without thrombocytopenia. We found that patients without thrombocytopenia had TPO concentrations comparable with the normal controls. In contrast, patients with thrombocytopenia had significantly lower TPO concentrations. We also found a significant correlation between TPO concentrations and platelet count in the whole group of cirrhotic patients. We assume that cirrhotic patients, in whom production of TPO in the liver is maintained despite the severe liver disease, could counteract the sequestration of platelets in the spleen and maintain normal platelet count. In contrast, patients with inadequate production of the cytokine inevitably develop thrombocytopenia. The recent findings of Schimodaira et al that serum TPO concentrations may decrease with deterioration of protein producing ability of the liver in cirrhotic patients are in keeping with our data. ${ }^{13}$ More recently Sezai et al have reported that serum TPO concentrations are associated with portal haemodynamics. ${ }^{19}$ They performed colour Doppler ultrasonography of the splenic vein and showed that patients with hepatofugal flow direction, which usually indicates progressive liver disease, had lower serum TPO concentrations.

The inability of cirrhotic patients to increase platelet production by the bone marrow in response to increased consumption has been reported previously as the main determinant of thrombocytopenia in a seminal study by Stein and Harker. ${ }^{3}$ We suggest that this is caused by inadequate TPO production. Another hypothesis that has been proposed to explain thrombocytopenia together with low TPO concentrations in patients with cirrhosis was through an ill defined consequence of hypersplenismthat is, the sequestration of platelets bound with TPO in the spleen. However, we found no correlation between the spleen volume and platelet count before OLT. This finding is consistent with previous reports that alleviation of portal hypertension either by portocaval anastomoses or TIPS has not had significant effects in reducing thrombocytopenia as it usually does not lead to a persistent increase in platelet count. ${ }^{4-7}$ In addition the spleen volume does not appear to affect serum TPO, as we found no correlation between spleen index and TPO concentrations. This is in keeping with a recent study reporting that splenectomy does not significantly alter the serum TPO concentration. ${ }^{13}$

In patients who underwent OLT, platelet count decreased further in the first days after transplantation as has been reported in previous studies. ${ }^{20}{ }^{21}$ At the same time TPO concentrations increased and reached a peak on day 5 after transplantation. The peak in TPO concentrations was reached just after the nadir in platelet count after transplantation. The increase in TPO concentrations was significant for the patients with pretransplantation thrombocytopenia. We also found an inverse correlation between peak TPO concentrations and nadir platelet count after OLT similar to that observed in states of myelosuppression accompanying chemotherapy or radiation. ${ }^{11}{ }^{22}$ The increase in TPO concentrations was followed by a subsequent rise in numbers of platelets and return to normal counts within four to six days after peak TPO. The time course between peak TPO and subsequent normal platelet count is the same as that reported in previous experimental and clinical studies of myelosuppression induced thrombocytopenia. ${ }^{112324}$ All these data provide strong evidence that restored TPO production accounts for the rapid normalisation of platelet counts after OLT. It is important to mention that this rapid resolution of thrombocytopenia after transplantation occurs in the context of increased activation and sequestration of thrombocytes in the graft during the first days after liver transplantation as has been shown recently. ${ }^{25}{ }^{26}$ This underlines the importance of the restitution of adequate TPO production.

Three previous series have reported a similar increase in TPO after orthotopic liver transplantation. ${ }^{16}{ }^{17}{ }^{27}$ However, neither of these studies evaluated the volume of the spleen in order to clarify its role in the thrombocytopenia of liver cirrhosis. We believe that our study provides firm data to substantiate the association 
of low TPO concentrations with low platelet counts in cirrhosis, but high TPO concentrations with low platelet counts after OLT when normal synthesis is restored.

Finally, we found that the restored TPO production after liver transplantation was independent of the allograft function as we found no correlation between peak TPO and cold ischaemia time, peak aminotransferase and bilirubin concentrations after transplantation, and the presence of histological rejection.

In conclusion, our findings provide evidence that inadequate TPO production seems to be the major cause for thrombocytopenia in liver cirrhosis, although increased splenic sequestration of platelets in the enlarged spleen may have an additional role. Orthotopic liver transplantation can readily restore TPO production and lead to a rapid resolution of thrombocytopenia, and shows the putative normal feedback mechanism for maintenance of platelet numbers.

1 Aster R. Pooling of platelets in the spleen: role in the pathogenesis of "hypersplenic" thrombocytopenia. f Clin Invest 1966;45:645-57.

2 Harker LA, Finch CA. Thrombokinetics in man. 7 Clin Invest 1969;48:963-74.

3 Stein S, Harker LA. Kinetic and functional studies of platelets, fibrinogen, and plasminogen in patients with hepatic cirrhosis. F Lab Clin Med 1982;99:217-30.

4 Mutchnick MG, Lerner E, Conn HO. Effect of portacaval anastomosis on hypersplenism. Dig Dis Sci 1980;25:92938 .

5 Soper NJ, Rikkers LF. Effect of operations for variceal hemorrhage on hypersplenism. Am f Surg 1982;144:700-3.

6 Sanyal AJ, Freedman AM, Purdum PP, et al. The hematologic consequences of transjugular intrahepatic portosystemic shunts. Hepatology 1996;23:32-9.

7 Alvarez OA, Lopera GA, Patel V, et al. Improvement of thrombocytopenia due to hypertension after transjugula intrahepatic portosystemic shunt placement in cirrhotic patients. Am 7 Gastroenterol 1996;91:134-7.

8 Yamaga K, Tzakis AG, Shimada M, et al. Reversal of hypersplenism following orthotopic liver transplantation. Ann Surg 1989;210:180-3.

9 de Sauvage FJ, Hass PE, Spencer SD, et al. Stimulation of megakaryocytopoiesis and thrombopoiesis by the $\mathrm{c}-\mathrm{Mpl}$ ligand. Nature 1994;369:533-8.

10 Lok S, Kaushansky K, Holly RD, et al. Cloning and expression of murine thrombopoietin CDNA and stimulation of sion of murine thrombopoietin CDNA and stimulation
platelet production in vivo. Nature 1994;369:565-8.
11 Nichol JL, Hokom MM, Horncohl A, et al. Megakaryocyte growth and development factor: analysis of in vitro effects on human megakaryocytopoiesis and endogenous serum levels during chemotherapy induced thrombocytopenia. $\mathcal{F}$ Clin Invest 1995;95:2973-8.

12 Shimada Y, Kato T, Ogami K, et al. Production of thrombopoietin (TPO) by rat hepatocytes and hepatoma cell lines. Exp Hematol 1995;23:1388-96.

13 Shimodaira S, Ishida F, Ichikawa $\mathrm{N}$, et al. Serum thrombopoietin (c-Mpl ligand) levels in patients with liver cirrhosis. Thromb Haemost 1996;76:545-8.

14 Akriviadis EA, Cohen SM, Chen DCP, et al. Thrombopoietin (TPO) levels are increased in patients with cirrhosis and do not appear to play a role in the pathogenesis of thrombocytopenia of cirrhosis [abstract]. Hepatology 1997; 26:184A.

15 Satoi J, Sanjyou Y, Takekuma A, et al. Serum thrombopoitin and hepatosplenomegaly in patients with chronic liver disease [abstract]. Hepatology 1997;26:206A.

16 Peck-Radosavljevic M, Zacherl J, Meng GY, et al. Is inadequate thrombopoietin production a major cause of thrombocytopenia in cirrhosis of the liver? F Hepatol 1997; 27:127-31.

17 Martin TG, Somberg KA, Meng GY, et al. Thrombopoietin levels in patients with cirrhosis before and after orthotopic liver transplantation. Ann Intern Med 1997;127:285-8.

18 Siemensma NP, Bathal PS, Penington DG. The effect of massive liver resection on platelet kinetics in the rat. $\mathcal{F} \mathrm{Lab}$ Clin Med 1975;86:817.

19 Sezai S, Kamisaka K, Ikegami F, et al. Regulation of hepatic thrombopoietin production by portal hemodynamics in liver cirrhosis. Am f Gastroenterol 1998;93:80-2.

20 Plevak DJ, Halma GA, Forstrom LA, et al. Thrombocytopenia after liver transplantation. Transplant Proc 1988;20: 630-3.

21 McCaughan GW, Herkes R, Powers B, et al. Thrombocytopenia post liver transplantation: correlations with preoperative platelet count, blood transfusion requirements, allograft function and outcome. F Hepatol 1992;16:16-22.

22 Levin J. Thrombopoietin-clinically realized? $N$ Engl f Med 1997:336:434-6.

23 Grossmann A, Lenox J, Ren HP, et al. Thrombopoietin accelerates platelet, red blood cells, and neutrophil recovery in myelosuppressed mice. Exp Hematol 1996;24: 1238-46.

24 Meng YG, Martin TG, Peterson ML, et al. Circulating thrombopoietin concentrations in thrombocytopenic patients including cancer patients following chemotherapy with or without peripheral blood progenitor cell transplantation. Br 7 Haematol 1996;95:535-41.

25 Porte RJ, Blauw E, Knot EAR, et al. Role of the donor liver in the origin of platelet disorders and hyperfibrinolysis in liver transplantation. F Hepatol 1994;21:592-600.

26 Peck-Radosavljevic M, Wichlas M, Tai Nin C, et al. Thrombopoietin deficiency, DIC, thrombocyte consumption, and thrombocytopenia in cirrhosis [abstract]. I Hepatol 1998; 28(suppl 1):77.

27 Richards EM, Alexander GJM, Nichol JL, et al. Serum thrombopoietin levels following orthotopic liver transplantation [letter]. Thromb Haemost 1997;78:1420-1. 